\title{
Ultrasound-guided superficial cervical plexus block under dexmedetomidine sedation versus general anesthesia for carotid endarterectomy: a retrospective pilot study
}

\author{
Wangseok Do', Ah-Reum Cho', Eun-Jung Kim², Hyae-Jin Kim', Eunsoo Kim', Heon-Jeong Lee ${ }^{1}$ \\ ${ }^{I}$ Department of Anesthesia and Pain Medicine, Pusan National University, School of Medicine, Busan; \\ ${ }^{2}$ Department of Dental Anesthesia and Pain Medicine, Pusan National University Dental Hospital, Dental Research Institute, Yangsan, Korea
}

Background: Carotid endarterectomy (CEA) has been performed under regional and general anesthesia (GA). The general anesthesia versus local anesthesia for carotid surgery study compared the two techniques and concluded that there was no difference in perioperative outcomes. However, since this trial, new sedative agents have been introduced and devices that improve the delivery of regional anesthesia (RA) have been developed. The primary purpose of this pilot study was to compare intraoperative hemodynamic stability and postoperative outcomes between GA and ultrasound-guided superficial cervical plexus block (UGSCPB) under dexmedetomidine sedation for CEA.

Methods: Medical records from 43 adult patients who underwent CEA were retrospectively reviewed, including 16 in the GA group and 27 in the RA group. GA was induced with propofol and maintained with sevoflurane. The UGSCPB was performed with ropivacaine under dexmedetomidine sedation. We compared the intraoperative requirement for vasoactive drugs, postoperative complications, pain scores using the numerical rating scale, and the duration of hospital stay.

Results: There was no difference between groups in the use of intraoperative antihypertensive drugs. However, intraoperative inotropic and vasopressor agents were more frequently required in the GA group $(p<0.0001)$. In the GA group, pain scores were significantly higher during the first $24 \mathrm{~h}$ after surgery $(p<$ 0.0001 between $0-6 \mathrm{~h}, p<0.004$ between $6-12 \mathrm{~h}$, and $p<0.001$ between $12-24 \mathrm{~h}$ ). The duration of hospital stay was significantly more in the GA group $(13.3 \pm 4.6$ days in the GA group vs. $8.5 \pm 2.4$ days in the RA group, $p<0.001)$.

Conclusion: In this pilot study, intraoperative hemodynamic stability and postoperative outcomes were better in the RA compared to the GA group.

Keywords: Carotid endarterectomy; Cervical plexus block; Dexmedetomidine; General anesthesia; Ultrasonography

Received: January 29, 2018, Revised: March 28, 2018

Accepted: April 9, 2018

Corresponding Author: Ah-Reum Cho, Department of Anesthesia and Pain Medicine, School of Medicine, Pusan National University, 179, Gudeok-ro, Seo-gu, Busan 49241, Korea

Tel: +82-51-240-7399, Fax: +82-51-242-7466

E-mail: archo@pusan.ac.kr

\section{INTRODUCTION}

Carotid endarterectomy (CEA) has been successfully performed under regional anesthesia (RA) and general anesthesia (GA). A large randomized multicenter study of general anesthesia versus local anesthesia for carotid surgery (GALA) was conducted to determine whether GA or RA resulted in the

Copyright (C) 2018 Yeungnam University College of Medicine

This is an Open Access article distributed under the terms of the Creative Commons Attribution Non-Commercial License (http://creativecommons.org/licenses/by-nc/4.0/) which permits unrestricted non-commercial use, distribution, and reproduction in any medium, provided the original work is properly cited. 
better outcomes, including the proportion of patients with stroke, myocardial infarction (MI), or death within 30 days after anesthesia; survival free of stroke, MI or death up to 1 year after anesthesia; length of stay in hospital and blood pressure manipulation [1]. The GALA study concluded that the anesthetic technique does not affect perioperative outcomes. However, the trial has been criticized due to the wide variability in methodology; general anesthetic techniques were compared with a variety of regional anesthetic techniques. In addition, clinical practice had changed over the 8-year trial period. Since this study, there have been improvements in regional anesthetic techniques, intraoperative sedation, and the use of ultrasound guidance for RA [2,3]. In the GALA trial, RA was performed using landmark-guided superficial or deep cervical plexus block, which has been replaced by ultrasound-guided block, a method that allows direct visualization of structures and injection of local anesthetic [3].

The $\alpha_{2}$-adrenoreceptor agonist sedative, dexmedetomidine, is widely used in anesthetic practice. The $\alpha_{2}$-adrenoreceptors mediate sedation, sympatholysis, inhibition of vasoconstriction, and antinociception. The advantage of dexmedetomidine is that it has minimal respiratory depression [4]. Moreover, compared to sedation with a combination of midazolam and propofol, dexmedetomidine is less likely to cause perioperative hypertension and tachycardia. It also decreases intraoperative and postoperative anxiety and the need for opioids for postoperative pain control [5]. However, perioperative hypotension and bradycardia can occur [6].

Although the GALA trial did suggest a tendency towards a better hemodynamic profile in the RA group, the trial did not focus on the use of sedatives or perioperative hemodynamic status. The purpose of this pilot study was to test the hypothesis that for CEA, intraoperative hemodynamic stability is improved using an ultrasound-guided superficial cervical plexus block (UGSCPB) with dexmedetomidine sedation compared to GA. The primary outcome of this study was the requirement for vasoactive drugs between the two groups. Postoperative hemodynamic stability, pain severity, and complications within 1 year were also compared.

\section{MATERIALS AND METHODS}

\section{Study design}

This study was approved by the Institutional Review Board and exempted from the need to obtain informed consent. We retrospectively reviewed the electronic medical records of adult patients (aged between 46 and 91 years) who underwent CEA from June 2012 to July 2015. We chose this period because a single surgeon performed all CEA procedures during this time. We excluded patients who underwent a combined surgical procedure with CEA or underwent emergency surgery. The anesthetic technique was routinely determined by the anesthesiologist and the surgeon depending on the clinical condition of the patient. Patients were divided into the GA and the RA groups.

\section{Anesthetic technique}

Anesthesia was performed according to hospital protocols. In both groups, intraoperative monitoring included electrocardiography, invasive blood pressure (BP) measured from the radial artery, pulse oximetry, bispectral index monitoring (BIS; Bispectral Index ${ }^{\mathrm{TM}}$, Aspect Medical System Inc., Norwood, MA, USA), and near-infrared spectroscopy (NIRS; INVOS 5100; Somanetics Corp., Troy, MI, USA). GA was induced with intravenous propofol $(1-2 \mathrm{mg} / \mathrm{kg})$, remifentanil $(0.1 \mu \mathrm{g} /$ $\mathrm{kg} / \mathrm{min})$, and rocuronium $(1.0 \mathrm{mg} / \mathrm{kg})$ and maintained with sevoflurane and remifentanil. Sevoflurane was titrated to a BIS score of 40-60. Remifentanil was titrated to maintain the mean arterial pressure at $80 \%$ of the baseline. Mechanical ventilation was carried out with an oxygen/air mixture (fraction of inspired oxygen, 0.5 ) to maintain the end-tidal carbon dioxide at $30-40 \mathrm{mmHg}$. RA was performed by UGSCPB. After negative aspiration, $8-12 \mathrm{~mL}$ of $0.375-0.5 \%$ ropivacaine was injected beneath the sternocleidomastoid muscle. The sternocleidomastoid muscle was directly visualized sonographically (Vivid i, GE Healthcare, Wauwatosa, WI, USA) using a 12L-RS, $5-13 \mathrm{MHz}$ linear probe. Prior to incision, a loading dose of dexmedetomidine $(1.0 \mu \mathrm{g} / \mathrm{kg})$ was administrated over 10 minutes, followed by a maintenance infusion at $0.5 \mu \mathrm{g} / \mathrm{kg} / \mathrm{h}$, and adjusted to maintain a BIS score of 60-80 (infusion rate: $0.1-1.0 \mu \mathrm{g} / \mathrm{kg} / \mathrm{h}$ ). Adequacy of the RA was not documented; however, lidocaine $(0.5 \%)$ administered by the surgeon to treat intraoperative pain is common practice in our institution. Hypertension and tachycardia were managed with diltiazem (5-10 mg), esmolol (10-20 mg), labetalol (10 mg), or nicardipine (1-2 mg). To treat hypotension and bradycardia, intravenous ephedrine ( $10 \mathrm{mg}$ bolus) or atropine ( $0.5 \mathrm{mg}$ bolus) was 
administered. Persisting hypotension and bradycardia were treated with a continuous infusion of dopamine, dobutamine, norepinephrine, or phenylephrine. Postoperative pain was managed by the surgeon; morphine, $3 \mathrm{mg}$, and ketorolac, 30 mg were administered according to the numerical rating scale (NRS) until the pain was controlled.

\section{Outcome variables}

Demographic data, including the American Society of Anesthesiologists physical status, age, sex, weight, height, and coexisting diseases were obtained from the electronic medical records. Details on surgery-related outcomes, including the side of surgery, duration of surgery, type of anesthesia, carotid artery cross-clamping, and the use of a shunt were also obtained. Hemodynamic instability was categorized into three groups: none, no intervention; mild, 1 or 2 doses of vasoactive medication; severe, $\geq 3$ doses or a continuous infusion of vasoactive medication. We identified the frequency and timing of vasoactive drugs administered during surgery: T1, during the administration of dexmedetomidine or after intubation; T2, at skin incision; T3, before carotid artery crossclamping; T4, after carotid artery cross-clamping; T5, at the completion of surgery. Hemodynamic profiles during the $24 \mathrm{~h}$ postoperative period and the duration of hospital stay were recorded. Postoperative pain was rated using the NRS and recorded at 0-6 h, 6-12 h, and 12-24 h after surgery. Major complications including cardiovascular, respiratory, and neurological complications within 1 year were also compared.

\section{Statistical analysis}

Data are presented as number (\%), mean \pm standard deviation, or median (interquartile range). All continuous variables were checked for normal distribution with a Q-Q plot and the Kolmogorov-Smirnov test. Continuous variables that were normally distributed were compared with the Student's t-test. The frequency of interventions for hemodynamic instability and complications were compared with the chi-square or the Fisher's exact test. Postoperative NRS was compared using two-way repeated measures ANOVA with Bonferroni correction. For Bonferroni correction, a $p$-value of less than 0.0083 $(0.05 / 6)$ was considered significant. For other statistical tests, a $p$-value of less than 0.05 was considered significant. All analyses were performed using SPSS version 18.0 (SPSS Inc., Chicago, IL, USA).

\section{RESULTS}

Forty-eight patients underwent CEA during the study period. Five patients were excluded as they had combined or emergency surgery. Of the remaining 43 patients, 16 received GA and 27 received RA (Fig. 1).

Demographic data were not different between the two groups (Table 1). In the RA group, no patient required conversion to GA and no local anesthetic-related adverse effects were noted. Duration of surgery, anesthesia, and carotid artery cross-clamping were longer, and a shunt was used more frequently in GA group (Table 1). The frequency of intervention for hypertension and tachycardia was similar between groups; however, inotropic and vasopressor agents were more frequently used in the GA group ( $p<0.0001$, Table 2$)$. In the RA group, an antihypertensive drug was required in 51.9\% of patients at the time of administration of the loading dose of dexmedetomidine. In the GA group, 50\% of patients required an antihypertensive drug after intubation and at the time of the skin incision. An infusion of inotropic or vasopressor agents was required in $75 \%$ of patients in the GA group. In contrast, $18.5 \%$ of patients in the RA group required inotropic or vasopressor agents (Fig. 2). Intervention for hypertension was more frequently required in the GA group at

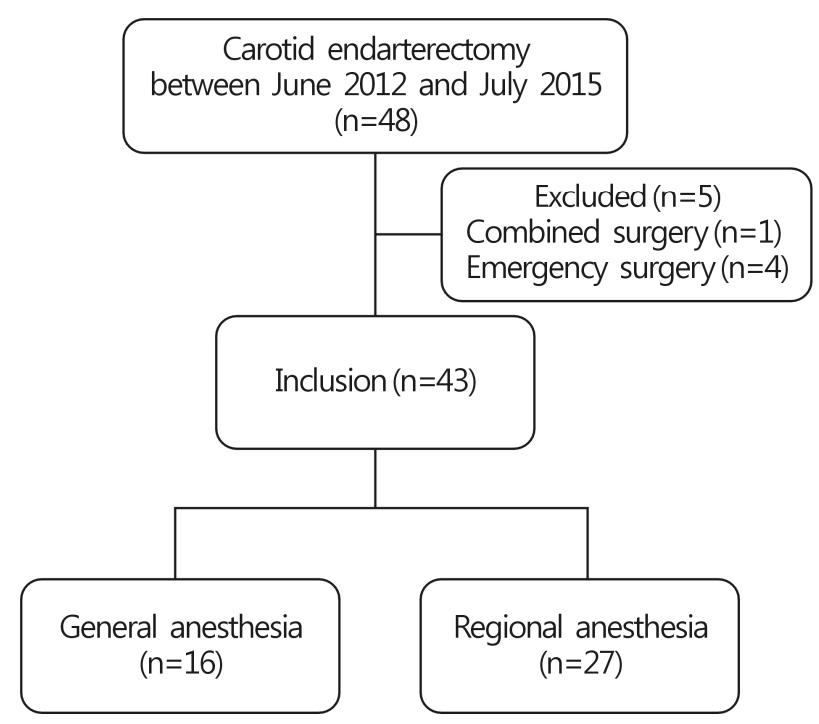

Fig. 1. Flow diagram of the patients. 
the time of skin incision (50\% in the GA group vs. $3.7 \%$ in the RA group, $p=0.001$ ) and at the completion of surgery
(31.3\% in the GA group vs. $3.7 \%$ in the RA group, $p=0.039$ ). Use of inotropic and vasopressor agents was significantly more

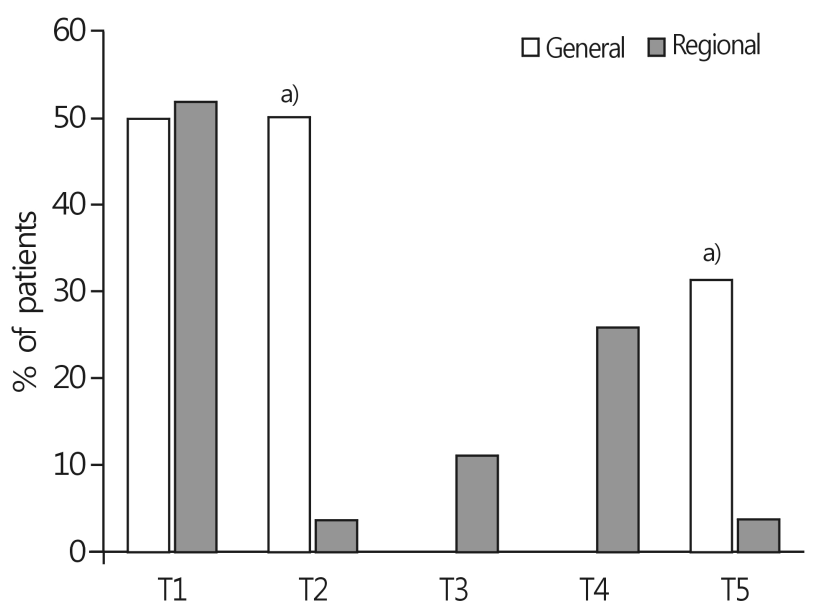
(A)

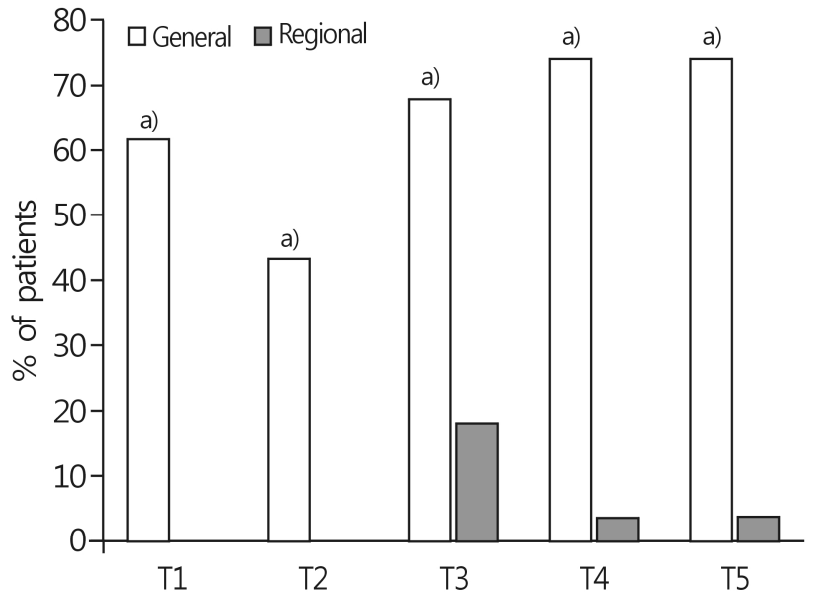

(B)

Fig. 2. Frequency and timing of antihypertensive drugs (A) and inotropic or vasopressors (B) administrating during surgery. T1, during administration of dexmedetomidine or after intubation; T2, at skin incision; T3, before carotid artery cross-clamping; T4, after carotid artery cross-clamping; T5, at the end of the operation. ${ }^{\text {a) }} p<0.005$ compared with regional anesthesia group.

Table 1. Demographic data and intraoperative outcomes

\begin{tabular}{lccc}
\hline \multicolumn{1}{c}{ Variable } & $\mathrm{GA}(\mathrm{n}=16)$ & $\mathrm{RA}(\mathrm{n}=27)$ & $p$-value \\
\hline ASA physical status (II/III) & $12(75) / 4(25)$ & $21(77.8) / 6(22.2)$ & 1.000 \\
Sex (male) & $14(87.5)$ & $23(85.2)$ & 1.000 \\
Age (year) & $72.7 \pm 8.2$ & $68.1 \pm 9.8$ & 0.124 \\
Weight (kg) & $62.9 \pm 9.0$ & $65.7 \pm 8.6$ & 0.318 \\
Height (cm) & $164.9 \pm 8.0$ & $164.7 \pm 5.4$ & 0.919 \\
Coexisting diseases & & & \\
Diabetes & $7(43.8)$ & $8(29.6)$ & 0.348 \\
Hypertension & $11(68.8)$ & $21(77.8)$ & 0.768 \\
Ischemic heart disease & $4(25.0)$ & $12(44.4)$ & 0.202 \\
Hyperlipidemia & $3(18.8)$ & $4(14.8)$ & 0.929 \\
TIA/stroke & $3(18.8)$ & $6(22.2)$ & 0.906 \\
COPD & $3(18.8)$ & $1(3.7)$ & 0.291 \\
Renal failure & $1(6.3)$ & $2(7.4)$ & 0.635 \\
Indication for surgery & & & 0.878 \\
Asymptomatic carotid stenosis & $1(6.3)$ & $4(14.8)$ & \\
TIA & $9(56.3)$ & $16(59.3)$ & \\
Stoke & $6(37.5)$ & $7(25.9)$ & \\
Location of surgery (rt) & $8(50.0)$ & $17(63.0)$ & 0.303 \\
Duration of surgery (min) & $147.8 \pm 29.6$ & $125.6 \pm 32.0$ & 0.029 \\
Duration of anesthesia (min) & $191.6 \pm 30.0$ & $164.1 \pm 29.4$ & 0.005 \\
Duration of clamping (min) & $49.1 \pm 10.4$ & $31.5 \pm 9.8$ & $<0.0001$ \\
Shunt & $15(93.8)$ & $5(18.5)$ & $<0.0001$ \\
\hline
\end{tabular}

Data are presented as mean $\pm \mathrm{SD}$ or number (\%).

ASA, American Society of Anesthesiologists; GA, general anesthesia; RA, regional anesthesia; TIA, transient ischemic attack; COPD, chronic obstructive pulmonary disease; Rt, right; SD, standard deviation. 
frequent in the GA group throughout the surgery (All $p<0.01$, Fig. 2).

Postoperative hemodynamic stability was not different between groups. Only $6.2 \%$ of patients in the GA group and $18.5 \%$ of patients in the RA group required intervention for hypotension, while $56.2 \%$ of patients in the GA group and $44.4 \%$ in the RA group received antihypertensive agents. NRS during the first 24 hours postoperatively was significantly higher $(p<0.0001$ between $0-6 \mathrm{~h}, p<0.004$ between $6-12$ $\mathrm{h}$, and $p<0.001$ between 12-24 h), and the duration of hospital stay was significantly longer ( $13.3 \pm 4.6$ vs. $8.5 \pm 2.4$ days, $p<0.001)$ in the GA group. The incidence of major complications was similar in both the groups (Table 3).

\section{DISCUSSION}

In this study, we found that intraoperative hemodynamic stability was better in the RA group compared to the GA group. The NRS during the first $24 \mathrm{~h}$ postoperatively and the duration of hospital stay were higher in the GA group. However, major postoperative complications within 1 year were not significantly different between groups.

RA has the advantage of ease of assessment of neurological status during carotid cross-clamping. RA has been in use for CEA for over 15 years. The GALA [1] and the other studies $[7,8]$ have not identified a statistically significant difference in the incidence of major perioperative outcomes, such as stroke, MI, or death between RA and GA. However, if major perioperative outcomes were similar between GA and RA, hemodynamic stability, postoperative pain, minor complications, and duration of hospital stay may be appropriate outcomes to determine the better anesthetic technique for CEA. Thus, well-standardized RA with advanced techniques needs to be compared with GA to evaluate perioperative outcomes.

Despite the ease of perioperative neurological assessment with RA, this technique has several risks. Systemic toxicity from local anesthetics can be a life-threatening complication resulting from intravascular injection or overdose [9]. Puncture-related complications include vertebral artery injection, subarachnoid or epidural injection, Horner's syndrome, phre-

Table 2. Hemodynamic stability during surgery

\begin{tabular}{lccc}
\hline \multicolumn{1}{c}{ Variable } & $\mathrm{GA}(\mathrm{n}=16)$ & $\mathrm{RA}(\mathrm{n}=27)$ & $p$-value \\
\hline Intervention for hypertension and tachycardia $(\mathrm{n})$ & & & 0.828 \\
None & $7(43.8)$ & $14(51.9)$ & \\
Mild & $6(37.5)$ & $11(40.7)$ & \\
Severe & $3(18.8)$ & $2(7.4)$ & $<0.0001$ \\
Intervention for hypotension and bradycardia (n) & & & \\
None & $1(6.2)$ & $22(81.5)$ & $4(14.8)$ \\
Mild & $3(18.8)$ & $1(3.7)$ & \\
Severe & $12(75.0)$ & & \\
Drugs to treat hypertension and tachycardia & & $16(59.3)$ & \\
Diltiazem & $5(31.3)$ & $1(3.7)$ & \\
Esmolol & $5(31.3)$ & $5(18.5)$ & \\
Nicardipine & $1(6.3)$ & $3(11.1)$ & \\
Labetalol & $0(0)$ & $2(7.4)$ & \\
Drugs to treat hypotension and bradycardia & & $2(7.4)$ & \\
Ephedrine & $9(56.3)$ & $1(3.7)$ & \\
Atropine & $0(0)$ & $0(0)$ \\
Dopamine & $9(56.3)$ & $0(0)$ \\
Dobutamine & $1(6.3)$ & $0(0)$ \\
Norepinephrine & $1(6.3)$ & \\
Phenylephrine & $3(18.8)$ & & \\
\hline
\end{tabular}

Data are presented as number (\%).

GA, general anesthesia; RA, regional anesthesia; n, number; None, no intervention; Mild, 1 or 2 injections of drugs; Severe, 3 injections of drugs or infusion. 
nic nerve block, and local hematomas [10]. Advantages of ultrasound-guided block over the landmark-based technique include the ability to control the depth of needle insertion and avoid inadvertent puncture of neighboring structures [11]. Ultrasonography is now commonly used for the visualization of real-time images when performing nerve blocks. The advantages of the ultrasound-guided technique over the landmark technique are the direct visualization of nerves and adjacent anatomical structures, observation of the needle and local anesthetic spread during injection, detection of anatomical abnormalities, and reduced volume of local anesthetic [12], leading to a lower incidence of RA-related complications and increased safety. In our study, superficial cervical plexus block was performed with $8-10 \mathrm{~mL}$ of ropivacaine $(0.375-0.5 \%)$, which is less than the $20-30 \mathrm{~mL}$ volume recommended with the landmark technique [13-15]. In the GALA study, 4.4\% of patients in the RA group had complications that led to the cancellation of surgery or conversion to GA [1]. The reasons for cancellation of surgery or conversion to GA were patient preference, problems with positioning the patient, deterioration of clinical condition after local-anesthetic block, pain at the operative site, general anxiety, physiological instability, difficult surgery, and neurological deterioration on cross-clamping [1]. In our study, however, no patient required conversion to GA. Precise identification of the superficial cervical plexus by ultrasonography results in the use of a smaller volume of local anesthetic compared to the landmark technique to provide an adequate nerve block for CEA.

Hemodynamic instability is very common during CEA due to impaired autoregulation of arterial pressure after a stroke and reduced baroreceptor sensitivity because of carotid atherosclerosis; besides, the effects of carotid surgery, anesthesia, age, diabetes, and antihypertensive medications may also lead to hemodynamic instability [16]. With the use of GA, patients are more likely to be hypotensive and generally require vasopressor support [17-19]. In the postoperative period, patients who undergo GA are likely to be hypertensive, due to emergence from anesthesia and possibly, pain. The perioperative course was similar in patients who underwent GA in our study.

On the other hand, patients receiving RA are often hypertensive during surgery, particularly during the cross-clamp period; however, they become hypotensive after surgery [20].

Table 3. Postoperative outcomes

\begin{tabular}{|c|c|c|c|}
\hline Variable & GA $(n=16)$ & $\mathrm{RA}(\mathrm{n}=27)$ & $p$-value \\
\hline \multicolumn{4}{|l|}{ Hemodynamic profiles } \\
\hline Intervention for hypertension and tachycardia (n) & & & 0.697 \\
\hline None & $7(43.8)$ & $15(55.6)$ & \\
\hline Mild & $4(25.0)$ & $8(29.6)$ & \\
\hline Severe & $5(31.2)$ & $4(14.8)$ & \\
\hline Intervention for hypotension and bradycardia (n) & & & 0.539 \\
\hline None & $15(93.8)$ & $22(81,5)$ & \\
\hline Mild & $0(0)$ & $4(14.8)$ & \\
\hline Severe & $1(6.2)$ & $1(3.7)$ & \\
\hline NRS & & & 0.007 \\
\hline $0-6 \mathrm{hr}$ & $6(5-8)$ & $4(2-5)$ & $<0.0001^{\text {a) }}$ \\
\hline $6-12 \mathrm{hr}$ & $5(3-5)$ & $2(0.5-3.5)$ & $0.004^{\mathrm{a})}$ \\
\hline $12-24 \mathrm{hr}$ & $3(2-3)$ & $2(0.5-2)$ & $0.001^{\mathrm{a})}$ \\
\hline \multicolumn{4}{|l|}{ Complications } \\
\hline Neurologic & $6(37.5)$ & $7(25.9)$ & 0.649 \\
\hline Cardiac & $1(6.2)$ & $2(7.4)$ & 0.635 \\
\hline Respiratory & $1(6.2)$ & $0(0)$ & 0.788 \\
\hline Hospital stay (day) & $13.3 \pm 4.6$ & $8.5 \pm 2.4$ & 0.001 \\
\hline
\end{tabular}

Data are presented as mean $\pm \mathrm{SD}$, median (interquartile range) or number (\%).

GA, general anesthesia; RA, regional anesthesia; NRS, n, number; numeric rating scale; SD, standard deviation; None, no intervention; Mild, 1 or 2 injections of drugs; Severe, 3 injections of drugs or infusion.

a) The significance level after Bonferroni correction is 0.0083 . 
These observations may be explained by preserved cerebral autoregulation when the patient is awake [21]. Local anesthesia inhibits carotid sinus nerve fibers beneath the carotid bifurcation and decreases baroreceptor sensitivity, leading to increased intraoperative sympathetic activity [22]. Additionally, the stressful, awake state and absence of GA-related cardiovascular depression might increase the incidence of hypertension and tachycardia [23]. However, except at T1, only 25.9\% of patients who underwent RA required intervention for hypertension and tachycardia in our study. This is likely due to dexmedetomidine infusion, which is associated with fewer interventions for the treatment for hypertension and tachycardia, and less postoperative pain compared with other sedatives [5,6]. In a previous study, $40 \%$ patients who received dexmedetomidine required treatment for hypertension and tachycardia, compared with $72 \%$ of patients who received fentanyl and midazolam sedation [6]. A concern in the RA group of our study was the hypertension observed during administration of the loading dose of dexmedetomidine, with $50 \%$ of patients requiring a vasodilator. Dexmedetomidine produces vasoconstriction by acting on peripheral $\alpha_{2 b}$-adrenoreceptors while simultaneously exerting sympatholytic effects by binding to central $\alpha_{2 a}$-adrenoreceptors [24]. In anesthetized subjects or subjects with reduced sympathetic nervous system tone, increasing doses of dexmedetomidine have a more prominent vasoconstrictor effect [25]. However, this can be easily managed with low doses of vasodilators, such as diltiazem (10 $\mathrm{mg}$ ) or nicardipine (1 mg).

The greatest advantage of RA during CEA is the continuous and direct feedback of brain function that it provides to the surgeon. During carotid clamping, assessment of brain function in the awake patient reliably alerts the surgeon to the need for a shunt. Since assessment of brain function could not be done in patients under GA, a shunt was placed more frequently, and intraoperative monitoring of brain function was done more often in patients in the GA group. The GALA trial demonstrated a $29 \%$ reduction in the use of a shunt with RA compared to GA [1]. In our study, shunts were used in 93.8\% patients receiving GA and $18.5 \%$ in patients with RA. Although the use of a shunt is common practice and should protect the brain from stroke [26,27], intraoperative shunt placement is a strong predictor of perioperative strokes [28].

Postoperative pain was significantly lower in the RA group in our study. The difference within the first 6 hours could be the combined effect of RA and dexmedetomidine. Additionally, patients in the GA group received a remifentanil infusion, which causes postoperative hyperalgesia [29]. Overall, postoperative complications occurred more frequently in patients who underwent GA, and major complications, including MI and acute asthma, occurred in the GA group. The duration of hospital stay was significantly longer in the GA (13.3 \pm 4.6 days) than in the RA group $(8.5 \pm 2.4$ days). Our results are similar to those of a recently published study [30], in which patients who underwent GA had a significantly higher odds ratio for postoperative MI, acute congestive heart failure, and hemodynamic instability compared to RA. Additionally, the GA group showed extended duration of hospital stay for more than one day in their study.

There are several limitations in this study. First, we included only 43 patients according to the post hoc power analysis with comparison of the occurrence of intraoperative hypotension between the GA and the RA groups as one of the primary outcomes [31]. However, our results should be interpreted cautiously. In particular, considering the low incidence of major complications after CEA, no definitive conclusions can be drawn. Second, the reason for the choice of anesthetic technique was not specified in the medical records, which might be a potential source of bias. The anesthetic technique was chosen according to the physical status and patient characteristics, preference of the surgeon and the anesthesiologist, which may have influenced the results of our study.

This retrospective pilot study suggests that for CEA, UGSCPB with dexmedetomidine sedation provides better intraoperative hemodynamic stability and more favorable postoperative outcomes compared to GA. Large prospective studies are needed in the future before conclusions can be drawn about the validity of our findings. In addition, a prospective randomized controlled trial should compare outcomes between well-standardized RA and GA.

\section{CONFLICT OF INTEREST}

No potential conflict of interest relevant to this article was reported.

\section{ORCID}

Wangseok Do, https://orcid.org/0000-0003-2338-9648 
Ah-Reum Cho, https://orcid.org/0000-0002-7108-1384

Eun-Jung Kim, https://orcid.org/0000-0003-4982-9517

Hyae-Jin Kim, https://orcid.org/0000-0003-1444-1125

Eunsoo Kim, https://orcid.org/0000-0001-9978-4973

Hyeon-Jeong Lee, https://orcid.org/0000-0002-3450-865X

\section{REFERENCES}

1. GALA Trial Collaborative Group; Lewis SC, Warlow CP, Bodenham AR, Colam B, Rothwell PM, et al. General anaesthesia versus local anaesthesia for carotid surgery (GALA): a multicentre, randomised controlled trial. Lancet 2008;372: 2132-42.

2. Guay J. Regional anesthesia for carotid surgery. Curr Opin Anaesthesiol 2008;21:638-44.

3. Martusevicius R, Swiatek F, Joergensen LG, Nielsen HB. Ultrasound-guided locoregional anaesthesia for carotid endarterectomy: a prospective observational study. Eur J Vasc Endovasc Surg 2012;44:27-30.

4. Sidorowicz M, Owczuk R, Kwiecińska B, Wujtewicz MA, Wojciechowski J, Wujtewicz M. Dexmedetomidine sedation for carotid endarterectomy. Anestezjol Intens Ter 2009;41: 78-83.

5. Bekker AY, Basile J, Gold M, Riles T, Adelman M, Cuff G, et al. Dexmedetomidine for awake carotid endarterectomy: efficacy, hemodynamic profile, and side effects. J Neurosurg Anesthesiol 2004;16:126-35.

6. McCutcheon CA, Orme RM, Scott DA, Davies MJ, McGlade DP. A comparison of dexmedetomidine versus conventional therapy for sedation and hemodynamic control during carotid endarterectomy performed under regional anesthesia. Anesth Analg 2006;102:668-75.

7. Tangkanakul C, Counsell CE, Warlow CP. Local versus general anaesthesia in carotid endarterectomy: a systematic review of the evidence. Eur J Vasc Endovasc Surg 1997;13:491-9.

8. Vaniyapong T, Chongruksut W, Rerkasem K. Local versus general anaesthesia for carotid endarterectomy. Cochrane Database Syst Rev 2013;(12):CD000126.

9. Lirk P, Picardi S, Hollmann MW. Local anaesthetics: 10 essentials. Eur J Anaesthesiol 2014;31:575-85.

10. Pandit JJ, Satya-Krishna R, Gration P. Superficial or deep cervical plexus block for carotid endarterectomy: a systematic review of complications. Br J Anaesth 2007;99:159-69.

11. Hadzic A. Hadzic's peripheral nerve blocks and anatomy for ultrasound-guided regional anesthesia. 2nd ed. New York: McGraw-Hill Medical; 2012. p. 346-51.

12. Marhofer P, Harrop-Griffiths W, Kettner SC, Kirchmair L. Fifteen years of ultrasound guidance in regional anaesthesia: part 1. Br J Anaesth 2010;104:538-46.

13. Demirel S, Celi de la Torre JA, Bruijnen H, Martin E, Popp E, Böckler D, et al. Effect of Superficial Cervical Plexus Block on Baroreceptor Sensitivity in Patients Undergoing Carotid Endarterectomy. J Cardiothorac Vasc Anesth 2016;30:30916.
14. Merle JC, Mazoit JX, Desgranges P, Abhay K, Rezaiguia S, Dhonneur $\mathrm{G}$, et al. A comparison of two techniques for cervical plexus blockade: evaluation of efficacy and systemic toxicity. Anesth Analg 1999;89:1366-70.

15. de Sousa AA, Filho MA, Faglione W Jr, Carvalho GT. Superficial vs combined cervical plexus block for carotid endarterectomy: a prospective, randomized study. Surg Neurol 2005;63(Suppl 1):S22-5.

16. Michenfelder JD, Sundt TM, Fode N, Sharbrough FW. Isoflurane when compared to enflurane and halothane decreases the frequency of cerebral ischemia during carotid endarterectomy. Anesthesiology 1987;67:336-40.

17. Umbrain V, Keeris J, D'Haese J, Verborgh C, Debing E, Van den Brande P, et al. Isoflurane, desflurane and sevoflurane for carotid endarterectomy. Anaesthesia 2000;55:1052-7.

18. Hartsell PA, Calligaro KD, Syrek JR, Dougherty MJ, Raviola CA. Postoperative blood pressure changes associated with cervical block versus general anesthesia following carotid endarterectomy. Ann Vasc Surg 1999;13:104-8.

19. Gassner M, Bauman Z, Parish S, Koenig C, Martin J, Hans S. Hemodynamic changes in patients undergoing carotid endarterectomy under cervical block and general anesthesia. Ann Vasc Surg 2014;28:1680-5.

20. McKevitt FM, Sivaguru A, Venables GS, Cleveland TJ, Gaines PA, Beard JD, et al. Effect of treatment of carotid artery stenosis on blood pressure: a comparison of hemodynamic disturbances after carotid endarterectomy and endovascular treatment. Stroke 2003;34:2576-81.

21. McCleary AJ, Dearden NM, Dickson DH, Watson A, Gough MJ. The differing effects of regional and general anaesthesia on cerebral metabolism during carotid endarterectomy. Eur J Vasc Endovasc Surg 1996;12:173-81.

22. Gottlieb A, Satariano-Hayden P, Schoenwald P, Ryckman J, Piedmonte M. The effects of carotid sinus nerve blockade on hemodynamic stability after carotid endarterectomy. J Cardiothorac Vasc Anesth 1997;11:67-71.

23. Hoefer J, Pierer E, Rantner B, Stadlbauer KH, Fraedrich G, Fritz J, et al. Ultrasound-guided regional anesthesia for carotid endarterectomy induces early hemodynamic and stress hormone changes. J Vasc Surg 2015;62:57-67.

24. Piascik MT, Soltis EE, Piascik MM, Macmillan LB. Alphaadrenoceptors and vascular regulation: molecular, pharmacologic and clinical correlates. Pharmacol Ther 1996;72:21541.

25. Ebert TJ, Hall JE, Barney JA, Uhrich TD, Colinco MD. The effects of increasing plasma concentrations of dexmedetomidine in humans. Anesthesiology 2000;93:382-94.

26. Jamil M, Usman R, Ghaffar S. Advantages of selective use of intraluminal shunt in carotid endarterectomy: a study of 122 cases. Ann Vasc Dis 2016;9:285-8.

27. Kong J, Li J, Ye Z, Fan X, Wen J, Zhang J, et al. Carotid endarterectomy with routine shunt for patients with contralateral carotid occlusion. Ann Thorac Cardiovasc Surg 2017;23: 227-32.

28. Khattar NK, Friedlander RM, Chaer RA, Avgerinos ED, Kretz ES, Balzer JR, et al. Perioperative stroke after carotid endar- 
terectomy: etiology and implications. Acta Neurochir (Wien) 2016;158:2377-83.

29. Shin SW, Cho AR, Lee HJ, Kim HJ, Byeon GJ, Yoon JW, et al. Maintenance anaesthetics during remifentanil-based anaesthesia might affect postoperative pain control after breast cancer surgery. Br J Anaesth 2010;105:661-7.

30. Dakour Aridi H, Paracha N, Nejim B, Locham S, Malas MB.
Anesthetic type and hospital outcomes after carotid endarterectomy from the Vascular Quality Initiative database. J Vasc Surg 2018;67:1419-28.

31. Faul F, Erdfelder E, Lang AG, Buchner A. G*Power 3: a flexible statistical power analysis program for the social, behavioral, and biomedical sciences. Behav Res Methods 2007;39: 175-91. 